\title{
Sensitivity Optimization of airflow level posture sensor
}

\author{
Duanlei $^{1, a^{*}, \text { Piao linhua }}{ }^{2, b}$, Dong haoliang ${ }^{3, c}$
}

(1Sensor Technique Research Center, Beijing Information Science \& Technology University, Beijing 100101,China;2 Beijing Key Laboratory for sensor, Beijing Information Science \& Technology University, Beijing 100101;3Sensor Technique Research Center, Beijing Information Science \& Technology University, Beijing 100101,China;a duanlei6688@yeah.net; b bjplh@163.com; )

Keywords: airflow level posture sensor; thermal power; environment temperature; sensitivity Abstract. In this paper, in order to improve the sensitivity of the sensor, using FLUENT software to simulate the temperature field of the closed cavity to find out the optimal thermal wire position. The distribution of temperature field in the closed body are calculated when the sensor is in different inclined angle, power and included angle. Analyzed the relationship of the sensor's sensitivity between the heating power and angle. The calculation results show that the heating power increases, the sensitivity of the sensor also increases, the optimal location of sensor also changes, it provides a theoretical basis for the optimization airflow level posture sensor sensitivity.

Airflow level posture sensor is a new type of tilt sensor which is made by the character of the natural convection gas in the enclosed cavity. The airflow level posture sensor sensitive mass is gas which quality is almost equal to zero, so relative to other tilt sensor this sensor has the advantages of high overload, short response time, low cost and so on. It has great application prospects in some areas, but the sensitivity is relatively low relate to the solid type, liquid type sensor, domestic development sensitivity of gas type level posture sensor cannot meet the needs of some commercial and military industry, thus to find reasonable thermal wire position distribution of sensitivity is very important to improve. Sensitivity is mainly two aspects of the design of the circuit and sensor internal structure design to decide. Circuit design has been more mature. This article mainly research one wire structure which is put forward by Fx Zhang, hope to take advantage of low cost, high efficiency simulation calculation method of the FLUENT to simulate the temperature field in the closed cavity, get the reasonable thermal wire position in order to improve the sensitivity of the sensor.

\section{The working principle of airflow level posture sensor}

The sealed cavity of airflow level posture sensor is axisymmetric, the diameter of the thermal resistor is far less than the radius of the cylinder. As shown n Fig.1As shown n Fig.1, the two dimension structure's heat source is simplified to a circle, acceleration of gravity loads on the Y axis, sealed cavity radius $r$ is set to $10 \mathrm{~mm}$, four walls keep radiating. The resistance value of heat resistance wire is the same, two wire structure thermal resistor R1, R2 are symmetrical about the origin, the spacing of $\mathrm{d}$ is $6 \sim 10 \mathrm{~mm}$. The heat $\mathrm{q}$ of three wire structure is in the origin of the coordinate axes. By symmetry, the two thermal wires are at the same temperature, the bridge output is zero at this time. When the sensor tilts, because of the effect of natural convection, the two thermal wires are in different temperature contours, and thus lead to two different thermal wire resistance changes, finally the circuit output a certain voltage, through the output voltage we can get the tilt angle.

As shown in Fig.2, the detection circuit airflow level posture sensor is a whole bridge of wheatstone bridge circuit, the two arm of detect bridge consists of two metal thermal resistor R1 and R2. The working principle is that when the sensor tilts a certain angle relative horizontal, the two thermistor position change relative in temperature field, make the two thermistor 
temperature one drop and another rise, the two metal thermistor resistance change, caused current change, a bridge becomes out of balance, the output $U$ is corresponding to the angle $\theta$.
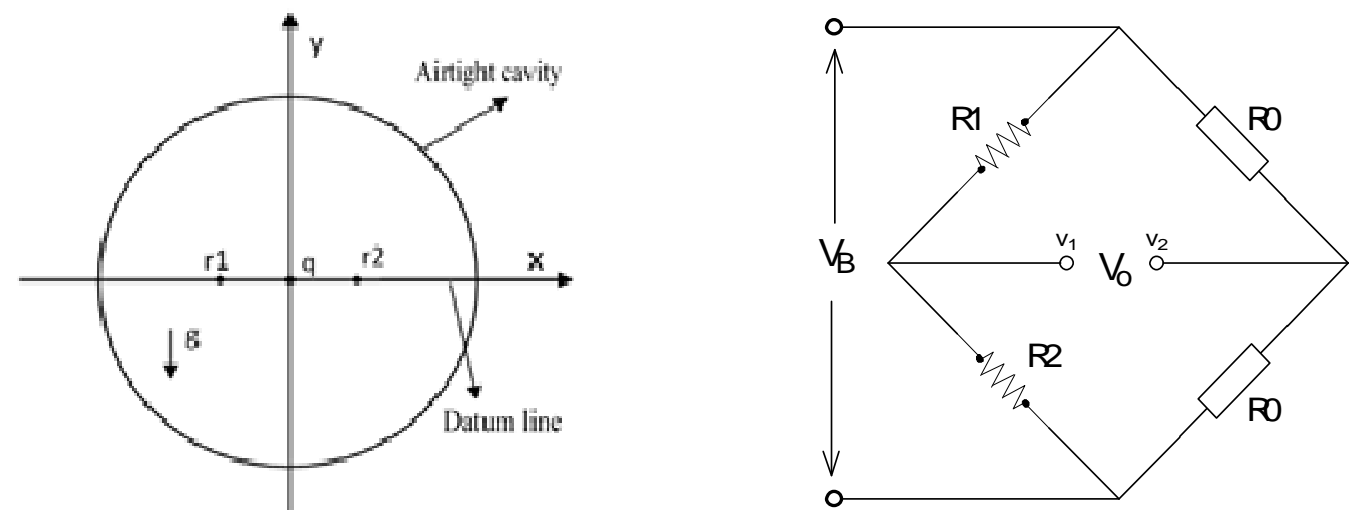

Fig.1 sensitive element simplified diagram Fig.2 Measure bridge circuit principle diagram

At this point, as shown in Fig.2 the bridge output can be represented as:

$$
\begin{gathered}
V_{0}=V_{B} \Delta \mathrm{R} / 2 \\
\Delta \mathrm{R}=\mathrm{R} 1-\mathrm{R} 2=\alpha_{1} \mathrm{R}_{0}\left(T_{a}-T_{b}\right)
\end{gathered}
$$

The $\Delta \mathrm{R}$ is resistance difference caused by the temperature difference on both thermistor. Put the (2) into (1), the relationship between the output voltage and the temperature difference can be obtained:

$$
V_{0}=V_{B} \alpha_{1} R(0)\left(T_{a}-T_{b}\right) / 2=V_{B} \alpha_{1} R(0) \Delta \mathrm{T} / 2
$$

After the temperature field of the gas in the sensor sealed cavity is fixed, the performance of the sensor depends on the thermistor resistance differential output. Seen from (3), and two thermistor sensor output voltage is proportional to the temperature difference of values, and setting a fixed temperature on the heat source is very big difference with setting a fixed power.

\section{FLUENT Solving}

FLUENT calculation results display the temperature contours graphically, then read and save the temperature of the corresponding position.

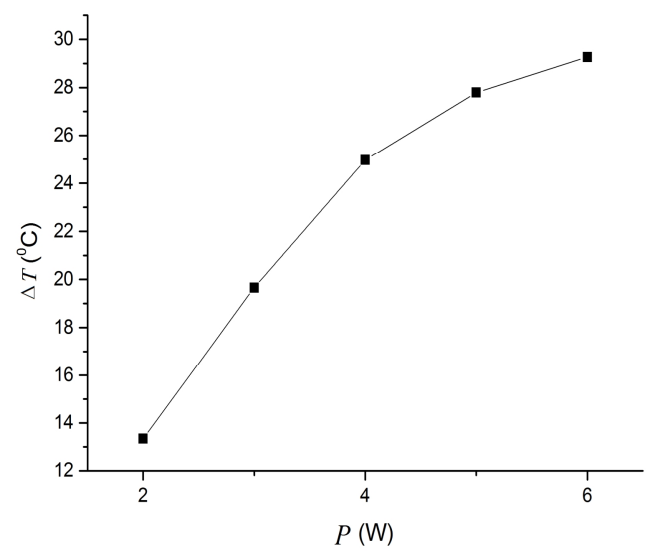

Fig.3 The relationship curve between the power and the temperature difference in different power 


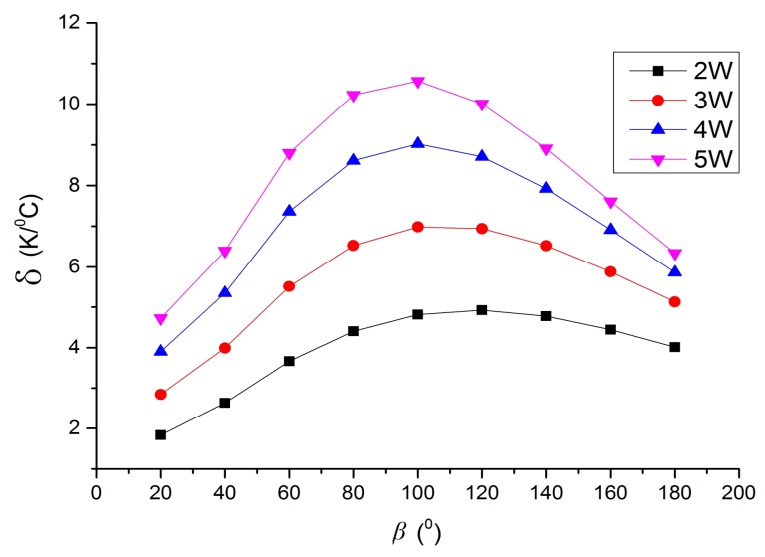

Fig.4 The sensitive change curve when the $\beta$ is different under the different power

Fig. 3 is the relationship curve between the power and the two thermal wire's temperature difference (the included angle $\beta$ is the angle between the two thermal wire and the center point). It can be seen from the diagram, the temperature field distribution redistribute under the different heating power. According to FLUET simulation results can be obtained that the role of buoyancy lift is more and more big, the trend of the air flow upward movement is more apparent, upward flow make the high temperature gas move towards the upper cavity and the gas temperature of circumferential homogeneity is more and more weak with the increase of power. Fig. 4 shows that with the increase of heating power, thermal wire induction to the temperature difference will increase. Thus, in the actual production, in the case of other conditions permit, increasing the heating wire power is an important way to increase the sensor sensitivity.

Fig.4 is the sensor's change curve respectively under the different heating power and different angle. From the curve in the graph it is easy to get the conclusion that increasing the heating power can make thermal wire optimal angle change, the optimal angle has the tendency to become smaller. Because with the increase of power, the high temperature of the gas will move up, the temperature gradient of the gas will also move. At the same time, comparing the curve in the graph can be seen that in the case of the same tilt angle, the heating wire power increasing obviously increase the sensitivity, when the angle is $100^{\circ}$, the sensitivity of power of $2 \mathrm{~W}$ is lower two times than $5 \mathrm{~W}$. So in actual production, in conditions allow, as far as possible to choose the high power heating wire to increase the sensitivity of the sensor.

\section{Conclusions}

Using the FLUENT software to simulate the temperature field in the closed cavity as to find out the optimal position of thermal wire in order to improve the sensitivity of the sensor. Respectively calculated the sensor's temperature field distribution in the different angle, power and angle in the closed cavity, analyzed the sensor sensitivity's relationship with the heating power and the angle. The calculation results show that the heating power increases, the sensitivity of the sensor also increases, the optimal location of sensor also change. While this article for the optimizing results is under certain conditions, but the method is universally applicable, in practice, according to the need to choose the appropriate power and thermal wire position to increase the sensitivity of the sensor, for pneumatic level posture sensor sensitivity optimization provides a theoretical basis. 


\section{Acknowledgement}

Beijing Natural Science Fund Project\&Beijing City Board of Education Science and technology key project(KZ201511232034); Key Laboratory of Beijing open projects funded project; Modern Control Technology funded by Ministry of Education Key Laboratory;

\section{Reference}

[1] Zx Chen. Pneumatic inertial sensor [D]. Ph. D. thesis, Huazhong University of science and technology, 1994

[2] Y Lin, Lh Piao, Fx Zhang. The structure principle of omnibearing gas pendulum inclination sensor [J]. Electronic components and materials. May 2006, 25(5):19-22 